\title{
Core-collapse SNe of type IIP and their progenitors: The case study of PNV J01315945+3328458
}

\author{
Raya Dastidar ${ }^{1 *}$, Brijesh Kumar $^{1}$, Devendra Kumar Sahu $^{2}$, Kuntal Misra ${ }^{1}$, \\ Mridweeka Singh ${ }^{1}$, Anjasha Gangopadhyay ${ }^{1}$, Gadiyara Chakrapani Anupama ${ }^{2}$, \\ Shashi Bhushan Pandey ${ }^{1}$.
}

${ }^{1}$ Aryabhatta Research Institute of observational sciencES, Nainital, 263002, India

${ }^{2}$ Indian Institute of Astrophysics, Koramangala, Bangalore, 560034, India

\begin{abstract}
The type II supernovae ( $\mathrm{SNe}$ ) are hydrogen-rich cosmic explosions resulting from the collapse of massive stars. The impetus of studying individual events arises from its cosmological importance and the diverse understanding of the evolution and explosion mechanism of such events. In this work, we present the preliminary photometric and spectroscopic analysis of a recent type IIP explosion, PNV J01315945+3328458 in the galaxy NGC 582. While the initial phases of these energetic events are bright enough to be observed with the 1-2m class telescopes, the supernovae fade below the detection limit of these telescopes in the nebular phase. In addition, the class of sub-luminous events with $\mathrm{Mv} \sim-15$ or the events occurring at higher redshift, fade below the detection limit of these telescopes very early in their evolution. Large aperture telescopes like the newly installed 3.6m Devasthal Optical Telescope (DOT) will ensure a longer coverage of such events and also to probe deeper into the Universe. With the $3.6 \mathrm{~m}$ telescope installed in Devasthal (DOT), we plan to study the progenitor environment of CCSNe to infer the metallicity at the explosion site.
\end{abstract}

\section{Introduction}

Massive stars typically in ZAMS mass range of 8-25 $\mathrm{M}_{\odot}$ (Heger et al. 2003; Eldridge and Tout 2004) end their stellar lives in a cosmic explosion leaving behind a neutron star as a compact remnant. The group of $\mathrm{SNe}$ which falls under this class and shows prominent hydrogen features in their spectra can be further classified into normal type IIP, exhibiting a plateau in their light curve and peculiar type IIP (SN 1987A like), showing a hump-like evolution in the corresponding plateau phase. The plateau phase of the light curve is powered by the energy deposited in the shell after shock breakout. The hydrogen layer ionized by the shock wave cools and recombines in this phase, allowing the trapped energy to be radiated away. PNV J01315945+3328458 is a normal type IIP event which exploded in the galaxy NGC 582. Monitoring campaigns were initiated soon after its discovery which was reported around 2015 Oct.02.92241 UT. Both photometric and spectroscopic observations were carried out with the 1-2m class telescopes in India.

\footnotetext{
*rayadastidar@aries.res.in
} 


\section{Observations and data reduction}

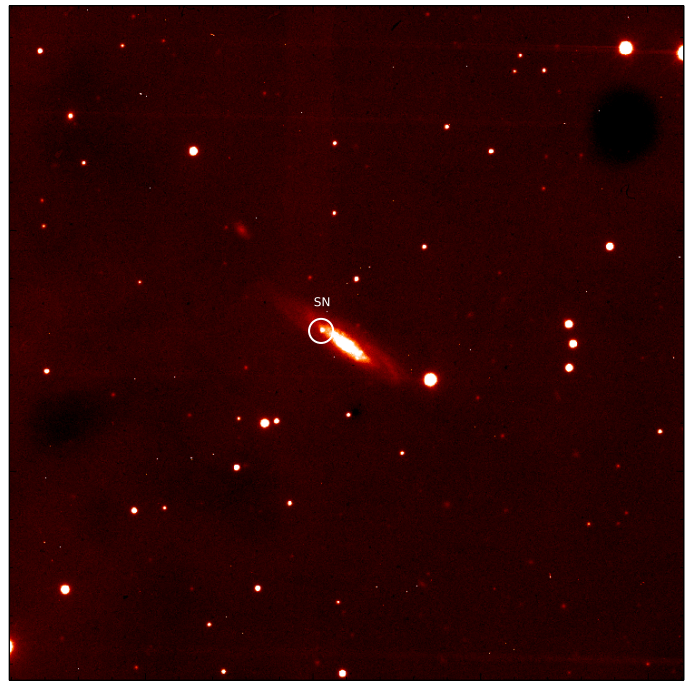

Figure 1: PNV J01315945+3328458 in NGC 582. $\mathrm{V}$ band image obtained with the $104 \mathrm{~cm}$ Sampurnanand Telescope, India.

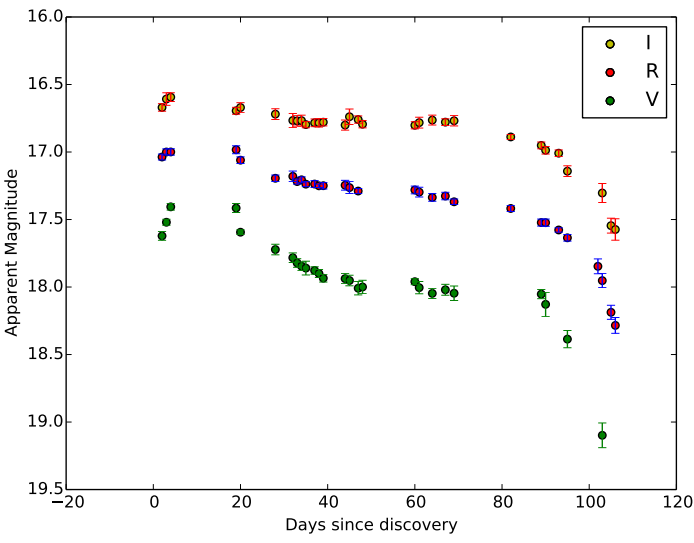

Figure 2: Light curve of PNV J01315945+3328458 in VRI bands.

We have used the ARIES $104 \mathrm{~cm}$ Sampurnanand Telescope (ST) and the $130 \mathrm{~cm}$ Devasthal Fast Optical Telescope (DFOT) for monitoring this event with the Johnson-Cousin BVRI filters. We followed this event up to 110 days post discovery. Standard tasks in IRAF were used for the preprocessing of the images. Owing to the location of the supernova in the host galaxy, PSF photometry was performed to eliminate the host galaxy contribution. We observed Landolt standard fields on 01 December 2015 along with the SN field to obtain the transformation coefficients from instrumental magnitude to standard magnitude. These transformation coefficients were used to generate a set of local standards in the SN field to perform differential photometry. Fig. 1 shows the location of the supernova in the host galaxy and in Fig. 2, we have presented the time evolution of the supernova through the V, R and I filters.

Study of individual SNe at various redshifts is necessary in order to constrain the parameters such as the ejecta velocity, the Ni-56 mass and the explosion energy of a particular class of events. However, a collection of good quality data of Type II SN is lacking as they evolve during some hundreds of days, making follow-up observations very time consuming. PNV J01315945+3328458 is a mere addition to such campaigns. Later, we plan to form a sample of type IIP SN to study the typical properties of this subclass.

\section{Preliminary results and discussion}

The results reported here are the following:

1. We have made a comparative study of the absolute V band light curve of PNV J01315945+3328458 with other type IIP supernovae (Fig. 3). It suggests that PNV J01315945+3328458 is most likely a normal type IIP event.

2. Valenti et al. (2016) made a comparative study of a sample of type IIP supernovae to find out the correlation between the various light curve parameters. They found a clear correlation between $\mathrm{s} 2$, the decline rate of the shallower slope of the light curve and the length of the plateau. A rough estimate of the s2 parameter obtained from our light curve is -0.18 in mag/50 days and from the 
correlation, we find that the plateau length corresponding to this value is around 100 days. However, in our case, the plateau length is nearly 90 days.

3. We could not continue the observation of this event post 110 days as the event went behind the sun and when it reappeared in the night sky, the magnitude had gone below the detection limit of 1-2m class telescopes. This emphasizes the possible contribution of the 3.6m DOT in our field.

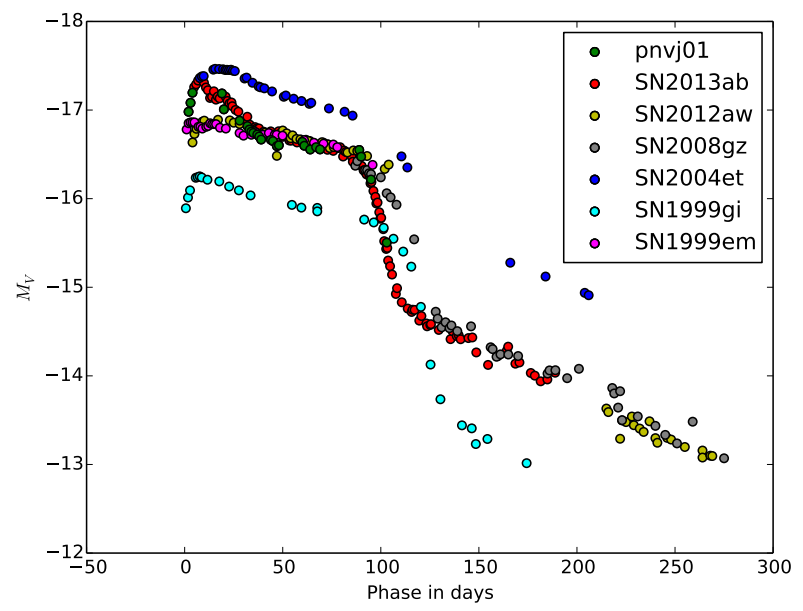

Figure 3: Comparison of the absolute V-band light curve of PNV J01315945+3328458 with those of other type IIP SNe like SN 1999em (Leonard et al. 2002), SN 1999gi (Leonard et al. 2002), SN 2004et (Sahu et al. 2006), SN 2008gz (Roy et al. 2011), SN 2012aw (Bose et al. 2013) and SN 2013ab (Bose et al. 2015). The magnitudes are distance and redshift corrected. The absolute V band magnitude of PNV J01315945+3328458 falls over other normal type IIP events indicating that it is most likely a normal type IIP event.

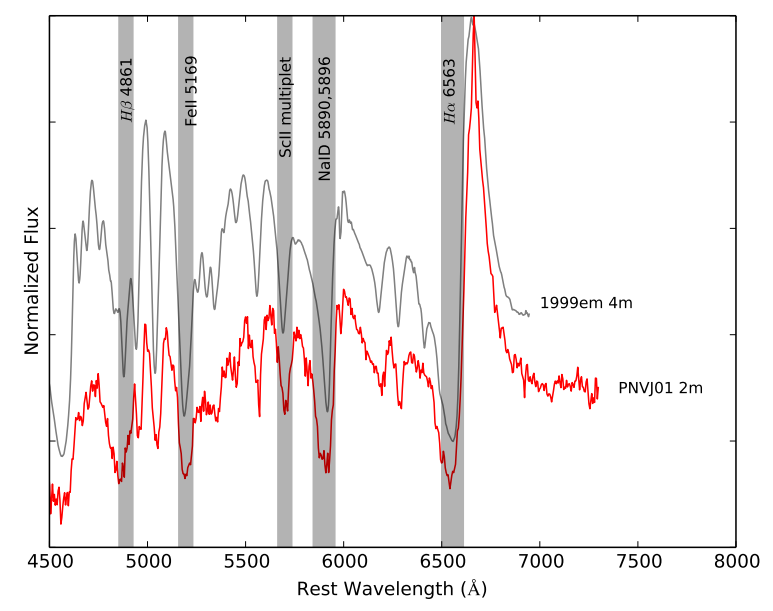

Figure 4: Comparison of the spectrum of SN 1999em (WiseRep) obtained with a $4 \mathrm{~m}$ class telescope with the spectrum of PNV J01315945+3328458 obtained with a $2 \mathrm{~m}$ class telescope at similar epoch. The spectrum obtained with a $4 \mathrm{~m}$ class telescope shows enhanced and more prominent spectral features while that obtained with a $2 \mathrm{~m}$ class telescope exhibits more line blending.

\section{Scope of study with the 3.6m Devasthal Optical Telescope}

Most of the cosmic explosions that are considered for the study comes from surveys which are partial towards brighter events. This leads to a bias in the observational results as we mostly ignore their fainter cousins. With the help of the $3.6 \mathrm{~m}$ DOT, we expect to have a complete sample by considering the low-luminosity events from the untargeted surveys.

Also, the nebular phase magnitude of most SNe reaches below the detection limit of 1-2m class telescopes. With the $3.6 \mathrm{~m}$ DOT, we can carry out deep nebular phase studies of these events using optical filters up to $25 \mathrm{mag}$ in a 300s exposure with a good signal-to-noise ratio.

Moreover, it is apparent that bigger aperture telescopes will enhance the resolution of the spectra. In Fig. 4, we have compared spectra obtained with $2 \mathrm{~m}$ and $4 \mathrm{~m}$ class telescopes. There is clearly less blending of lines and more prominent features in the spectra obtained with a $4 \mathrm{~m}$ class telescope. The higher resolution spectra will also allow the study of high velocity components of lines arising from circumstellar interaction which often gets blended with the normal component of these lines in low resolution spectra. 


\section{Acknowledgements}

We are thankful to the observing staff of $1.04 \mathrm{~m} \mathrm{ST,} 1.3 \mathrm{~m}$ DFOT and $2.01 \mathrm{~m}$ HCT for their kind support and cooperation.

\section{References}

Bose S., Kumar B., Sutaria F. et al. 2013, MNRAS, 433, 1871

Bose S., Valenti S., Misra K. et al. 2015, MNRAS, 450, 2373

Eldridge J. J., Tout C. A. 2004, MNRAS, 353, 87

Heger A., Fryer C. L., Woosley S. E., Langer N., Hartmann D. H. 2003, ApJ, 591, 288

Leonard, D. C., Filippenko, A. V., Alexei V. et al. 2002, PASP, 114, 35

Roy R., Kumar B. 2011, ApJ, 736, 76

Sahu D. K., Anupama G. C., Srividya S., Muneer S. 2006, MNRAS, 372, 1315

Valenti, S., Howell, D. A., Stritzinger, M. D. et al. 2016, MNRAS, 459, 3939 